\title{
Paradoxical saints: Polyvocality in an interactive AR digital narrative
}

\author{
Keywords \\ polyvocality, interactive digital narrative, syncretic illustration, magical realism, AR technology.
}

This artistic, practice-led $\mathrm{PhD}$ thesis is concerned with the potentials of polyvocality and interactive digital narrative. The practical project, Saints of Paradox, is constructed as a printed picture book that can be experienced through an Augmented Reality [AR] platform. The fictional story entails a woman who mourns the disappearance of her lover in the 1964 Brazilian coup d'état and lives for 40 years in a room of accumulated memories. IIn each illustration, the user can select three buttons on the tablet device that activates a different version of the story. Three narrators (saints) present interconnected but diverging interpretations of the events shaped by their distinct theological positions. The respective values of compassion, orthodoxy, and pragmatic realism distort details of imagery, sound, movement, and meaning. AR animated vignettes, each backed by a uniquely composed cinematic soundscape, allow characters to populate the luxuriously illustrated world. Candles flicker and burn, snakes curl through breathing flowerbeds, and rooms furnished with the contents of accumulated memories pulsate with mystery. The scanned image reviews an interactive parallax that produces a sense of threedimensional space, functioning as a technical and conceptual component. Theoretically, the story navigates relationships between the real and the imagined and refers to magical real binary modes of textual representation (Flores, 1955, Champi, 1980; Slemon, 1988, 1995; Spindler, 1993; Zamora and Faris; 1995; Bowers, 2004). Here, meaning negotiates an unreliable, sometimes paradoxical pathway between rational and irrational accounting and polyvocal narration. The dynamics between the book and the AR environments produce a sense of mixed reality (actual and virtual). The narrative experience resides primarily in an unstable virtual world, and the printed book functions as an enigmatic unoccupied vessel. Because of this, we encounter a sense of ontological reversal where the 'virtual' answers the ambiguities presented by the 'real' (the book). In the work, religious syncretism operates as a reference to Brazilian culture and an artistic device used to communicate a negotiation of different voices and points of view. The strange and somehow congruous forms of European, African, and indigenous influences merge to form the photomontage world of the novel. Fragments of imagery may be considered semiotic markers of cultural and ideological miscegenation and assembled into an ambiguous 'new real' state of being that suggests syncretic completeness. Methodologically, the project emanates from a post-positivist, artistic research paradigm (Klein, 2010). It is supported by a heuristic approach (Douglass \& Moustakas, 1985) to the discovery and refinement of ideas through indwelling and explicitness. Thus, the research draws upon tacit and explicit knowledge in developing a fictional narrative, structure, and stylistic treatments. A series of research methods were employed to assess the communicative potential of the work. Collaboration with other practitioners enabled high expertise levels and provided an informed platform of exchange and idea progression. 